\title{
In Tuberculosis -Uncommon Presentation of a Common Disease
}

\author{
Ashraf MU*, Zaheer MS and Rabbani MU \\ Department of Medicine JN Medical College, AMU Aligarh, UP, India \\ *Corresponding author: Muhammad Uwais Ashraf, Department of Medicine JN Medical College, AMU Aligarh, UP, India, Tel: 09760159391; E-mail: \\ uwaisashraf@gmail.com
}

Rec date: Apr 20, 2014, Acc date: Apr 28, 2014, Pub date: May 02, 2014

Copyright: @ 2014 Ashraf MU, et al. This is an open-access article distributed under the terms of the Creative Commons Attribution License, which permits unrestricted use, distribution, and reproduction in any medium, provided the original author and source are credited

\begin{abstract}
We present here a case report of a 27 year old man, who presented with high grade fever and swelling in the neck region. Peripheral blood smear showed both normocytic normochomic as well as microcytic hypochromic blood picture. Hemoglobin was $6.2 \mathrm{~g} \%$, TLC was 2100/ $\mathrm{mm}^{3}$, DLC was: L65, N30, E05, platelet count was 69000 (i.e. pancytopenia was documented). FNAC of the cervical lymph nodes revealed reactive hyperplasia, with no evidence of caseating or non-caseating granulomas. A differential diagnosis of aplastic anemia was made. However, bone marrow examination revealed acid fast bacilli (AFB) on ZN staining. A revised diagnosis of bone marrow tuberculosis was made and the patient was put on ATT. There was a dramatic response clinically and the pancytopenia reverted after treatment.
\end{abstract}

Keywords Tuberculosis; Pancytopenia; Autoimmune Haemolytic Anemia; IV immunoglobulin

\section{Introduction}

Tuberculosis is a common disease in developing countries and has diverse clinical manifestations. Patients can have various hematological abnormalities, most common being normocytic normochromic anemia, i.e. anemia of chronic disease [1]. Anemia in tuberculosis is most often due to nutritional deficiency, malabsorption syndromes, failure of iron utilization, and bone marrow suppression [2]. AIHA (autoimmune haemolytic anemia) is exceedingly rare in tuberculosis. Tuberculosis rarely has pancytopenia, and if present, it is found in miliary tuberculosis [3]. Coomb's positive hemolytic anemia is a very rare cause of anemia associated with TB and only few case reports have been published [4]. Apart from autoimmune hemolytic anemia, other autoimmune phenomena like vasculitis and thrombocytopenia have been associated with tuberculosis. Borie [5] reported a case series of 5 patients with tuberculosis and thrombocytopenia who were treated with ATT and other drugs like danazol, vincristine, IV immunoglobulin, and steroids [5]. We describe here the case of an immunocompetent patient who presented with fever of unknown origin and hematological derangements. The case presented here had tuberculous lymphadenitis associated with pancytopenia which resolved on treatment with anti tuberculous drugs and ancillary supportive therapy. The purpose of this paper is to draw attention to the importance of correlating the uncommon presentation of a commonly encountered condition and highlighting the fact that the prognosis of bone marrow TB depends largely on thorough intervention, timely diagnosis, rapid initiation of treatment and a thorough and timely follow up.

\section{Case Report}

Mr. Jeetu, a 27 year old male resident of Chirag Delhi presented in outpatient department of govt. medical college Mewat, Haryana with high grade fever and swelling in neck region from 2 weeks. There was no history of chills and rigors, hemoptysis, pain abdomen, nausea and vomiting, burning micturition, any bony pain, jaundice, hematemesis, abnormal swelling over any other part of the body, headache and seizures. There was also no past history of diabetes mellitus, hypertension, tuberculosis, exposure to unimmunized pets etc. Family history not significant. Patient was a non-smoker and office attendant by occupation.

On examination, the patients' pulse rate was 90 per minute, blood pressure was $110 / 70 \mathrm{mmHg}$, temperature was $101^{\circ} \mathrm{F}$; respiratory rate was 18 per minute. Pallor was present, there was no icterus, no cyanosis. Jugular Venous Pulse was not raised, there was no pedal edema. Bilateral cervical lymph nodes were enlarged, non-tender, mobile, rubbery and were not matted. Fundus examination revealed bilateral retinal vein occlusion with macular hemorrhage. On systemic examination, there was mild hepatosloenomegaly, chest was bilaterally clear and the cardiovascular examination was normal.

On investigation, hemoglobin was $6.2 \mathrm{~g} \%$, TLC was $2100 / \mathrm{mm} 3$, DLC was: L65, N30, E05, platelet count was 69000, peripheral blood smear revealed both normocytic normochomic as well as microcytic hypochromic blood picture, Reticulocyte count was $2.3 \%$ of RBC, LFT was normal but Alkaline phosphatase was raised by 2 times, Kidney function test was normal, Urine examination showed normal study, sputum for $\mathrm{AFB}$ was negative, Widal test was negative, Malaria serology was negative, chest $\mathrm{x}$-ray was normal, ECG was normal, USG Abdomen revealed hepatosplenomegaly, Serum electrophoresis for M band was negative, $24 \mathrm{hr}$ urinary calcium and serum calcium were also normal, HIV1 and 2 were negative. HBsAg and HCV were also negative, Vit. B12 levels were normal, leukocyte alkaline phosphatase was normal, Mantoux test was positive (18 $\mathrm{mm}$ induration), Coomb'stest was also negative. FNAC cervical lymph nodes revealed florid reactive hyperplasia. Lymph node biopsy showed no granulomatus lesion or caesious necrosis. Bone marrow examination showed: Polymorphs: $4 \%$, lymphocytes: $31 \%$, Eosinophils: $4 \%$, blasts $1 \%$, myelocytes: $22 \%$, metameylocytes: $15 \%$,Plasma cells: $15 \%$, erythroid series: $8 \%$. The overall impression of the bone marrow 
examination was: reactive marrow with plasmacytosis. However, on $\mathrm{ZN}$ staining of the marrow, acid fast bacilli (AFB) were seen (Figure 1).

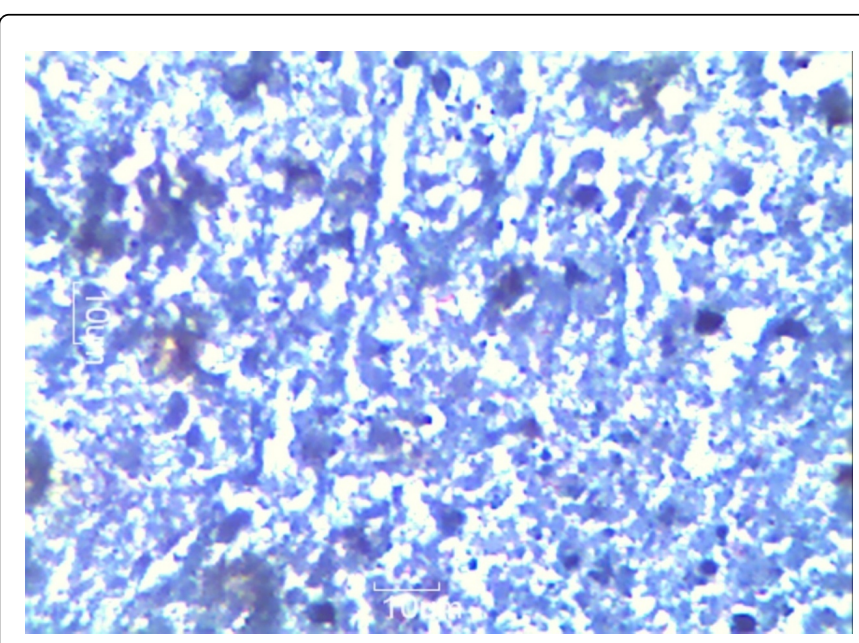

Figure 1: Mone marrow examination of the patient, showing acid fast bacilli on $\mathrm{ZN}$ staining

Based on the peripheral blood smear reports, the patient was earlier being treated as a case of aplastic anemia, until the AFB were demonstrated in the bone marrow. A diagnosis of disseminated tuberculosis was made and the patient was put on anti-tuberculous treatment. There was a dramatic response to treatment and there was improvement in clinical condition as well as reversal of pancytopenia.

\section{Discussion}

Tuberculosis is one of the oldest and most commonly encountered diseases. Although there is a significant and steady decline in the incidence of active pulmonary tuberculosis due to early diagnosis and prompt treatment, the incidence of extrapulmonary TB has remained constant particularly due to a delay in recognizing the condition when the presenting clinical scenario consists mostly of nonspecific extrapulmonary symptoms [6]. The hematologic manifestations in patients with miliary or disseminated tuberculosis include anemia, granulocytosis, leucopenia, leucocytosis, thrombocytosis and monocytosis $[7,8]$.

Pancytopenia as the presenting feature of disseminated tuberculosis is extremely rare both in children [9,10] as well as in adults [7]. Similar reports of tuberculosis induced pancytopenia are scarce in literature. The occurrence of pancytopenia in disseminated tuberculosis may well be attributed to hypersplenism [11], histiocytic hyperplasia and indiscriminate phagocytosis of blood cells by histiocytes in bone marrow [12], concomitant malnutrition [10] or infiltration of the bone marrow by caseating or noncaseating tuberculous granulomas [13].

Apart from infiltration of the marrow by the tuberculous granuloma, pancytopenia may also be caused by suppression of the bone marrow through release of interferon and lymphotoxin [14]. However, despite presence of tuberculous granulomas in a high proportion of patients with disseminated tuberculosis, pancytopenia is uncommon [15]. In our case the diagnosis of disseminated tuberculosis was established on clinical features, positive Mantoux test, cervical lymphadenopathy and demonstration of acid fast bacilli in the bone marrow. The peripheral blood picture became normal following anti-tuberculous therapy, thus supporting the diagnosis of tuberculosis induced pancytopenia. The other causes of pancytopenia including leukemia, chemicals, radiation, drugs, aplastic anemia and granulomas secondary to leprosy, syphilis, sarcoidosis and brucellosis were excluded on clinical and laboratory evaluation. This case suggests that disseminated tuberculosis may be considered as a cause of reversible pancytopenia.

Patients presenting with tuberculosis induced pancytopenia have a high mortality, but there are a few that have recovered also [16]. Documentation of the survivors is not ideal because isolation of the acid-fast bacilli is often difficult. In some cases bacteriological proof of mycobacteria is lacking and in others follow up was inadequate. The incidence of bone marrow granuloma ranges from $0.38 \%$ to $2.2 \%$ [17]. In contrast to good prognosis of pulmonary $\mathrm{TB}$, the literature review of various similar reported cases of bone marrow TB has revealed high mortality in the range of fifty to almost hundred percent.

Certain factors are thought to contribute to the variable outcome such as disease severity, other underlying pathologies leading to immunocompromised state, immunosuppressive therapies, and delay in initiation of appropriate treatment [18]. A retrospective chart review of bone marrow TB was done during the period from 1990 to 2002 at King Faisal Specialist Hospital and Research Center, Riyadh, which showed a mortality rate of 50\% [19]. The high mortality in that study was attributed to the delay in presentation [19]. Our patient showed good subjective response with subsidence of symptoms within few weeks following the initiation of the treatment. The favorable outcome in our patient is thought to be due to an early diagnosis, rapid start of treatment, good compliance to anti- TB medication, and thorough follow up.

A similar case has been reported by Alghamdi et al. [20]. They have reported a case of fever for six months with dizziness, fatigability and cough. However, their study had revealed typical caseating granulomas in the bone marrow apart from peripheral blood smear finding of pancytopenia. In yet another study by Singh et al., way back in 2001, the significance of hematological manifestations in tuberculosis has been highlighted [21]. They studied 23 patients of pulmonary tuberculosis and found that pancytopenia was present only in patients with disseminated and military tuberculosis $(\mathrm{p}<0.05)$.

Our case is unique because we have not demonstrated any granuloma in the bone marrow as compared to previous studies mentioned above. Another explanation of the hematological findings in our patient could be the presence of Macrophage Activation System (MAS). MAS, also known as Hemophagocytosis, is a non-specific clinical syndrome, which includes pancytopenia, hypertriglyceridemia and hyperferritinemia [22]. It is important to keep MAS in consideration because it is associated with a worse prognosis.

\section{Conclusion}

Tuberculosis is one of the common infections in this part of the world affecting the most productive age group. Hematological investigations are one of the commonly performed investigations in clinical practice. The varied hematological findings observed in tuberculosis should prompt us to consider tuberculosis as one of the differential diagnosis in patients with hematological findings. 
Citation: Ashraf MU, Zaheer MS, Rabbani MU (2014) In Tuberculosis -Uncommon Presentation of a Common Disease. J Clin Case Rep 4: 360. doi:10.4172/2165-7920.1000360

Page 3 of 3

\section{References}

1. Morris C, Bird AR, Nell H (1989) The haematological and biochemical changes in severe pulmonary tuberculosis. Q J Med 73: 1151-1159.

2. Glasser RM, Walker RI, Herion JC (1970) The significance of hematologic abnormalities in patients with tuberculosis. Arch Intern Med 125: 691-695.

3. Hunt BJ, Andrews V, Pettingale KW (1987) The significance of pancytopenia in miliary tuberculosis. Postgrad Med J 63: 801-804.

4. Nandennavar M, Cyriac S, Krishnakumar, Sagar T (2011) Immune hemolytic anemia in a patient with tuberculous lymphadenitis. J Glob Infect Dis 3: 89-91.

5. Raphael Borie,Claire Fieschi,Eric Oksenhendler, Lionel Galicier (2009) Tuberculosis associated thrombocytopenic purpura: effectiveness of antituberculous therapy. Hematol Rev. 1: e3.

6. Qasim ZA, Sarwari AR, Jilani SM (2003) Treatment failure of tuberculosis due to concomitant pathology. J Pak Med Assoc 53: 367-369.

7. Maartens G, Willcox PA, Benatar SR (1990) Miliary tuberculosis: rapid diagnosis, hematologic abnormalities, and outcome in 109 treated adults. Am J Med 89: 291-296.

8. Schuit KE (1979) Miliary tuberculosis in children. Clinical and laboratory manifestation in 19 patients. Am J Dis Child 133: 583-585.

9. Chandra J, Marwaha RK, Marwaha N, Kumar A, Mothanty D (1986) Pancytopenia and leukemoid reactions in tuberculosis. Indian J Pediatr 53: 665-667.

10. Kashyap S, Puri DS, Bansal SK, Dhawan A, Prasher N, et al. (1991) Mycobacterium tuberculosis infection presenting as pancytopenia with hypocellular bone marrow. J Assoc Physicians India 39: 497-498.

11. Meredith HC Jr, Early JQ Becker W (1949) Tuberculous splenomegaly with the hypersplenism syndrome; a case report. Blood 4: 1367-1373, illust.
12. Weintraub M, Siegman-Igra Y, Josiphov J, Rahmani R, Liron M (1984) Histiocytic hemophagocytosis in miliary tuberculosis. Arch Intern Med 144: 2055-2056.

13. Bagby GC Jr, Gilbert DN (1981) Suppression of granulopoiesis by Tlymphocytes in two patients with disseminated mycobacterial infection. Ann Intern Med 94: 478-481.

14. Murphy M, Loudon R, Kobayashi M, Trinchieri G (1986) Gamma interferon and lymphotoxin, released by activated $\mathrm{T}$ cells, synergize to inhibit granulocyte/monocyte colony formation. J Exp Med 164: 263-279.

15. Prout S, Benatar SR (1980) Disseminated tuberculosis. A study of 62 cases. S Afr Med J 58: 835-842.

16. Medd WE, Hayhoe FG (1955) Tuberculous miliary necrosis with pancytopenia. Q J Med 24: 351-364.

17. Feng J, Zhang H, Zhong DR (2009) [A clinical analysis of 20 cases with bone marrow granulomas]. Zhonghua Nei Ke Za Zhi 48: 485-487.

18. Wang JY, Hsueh PR, Wang SK, Jan IS, Lee LN, et al. (2007) Disseminated tuberculosis: a 10-year experience in a medical center. Medicine (Baltimore) 86: 39-46.

19. Eid A, Carion W, Nystrom JS (1996) Differential diagnoses of bone marrow granuloma. West J Med 164: 510-515.

20. Alghamdi AA, Awan FS, Maniyar IH, Alghamdi NA (2013) Unusual manifestation of extrapulmonary tuberculosis. Case Rep Med 2013: 353798.

21. Singh KJ, Ahluwalia G, Sharma SK, Saxena R, Chaudhary VP, et al. (2001) Significance of haematological manifestations in patients with tuberculosis. J Assoc Physicians India 49: 788, 790-794.

22. Le Hô H, Barbarot N, Desrues B (2010) [Pancytopenia in disseminated tuberculosis: Think of macrophage activation syndrome]. Rev Mal Respir 27: $257-260$ 